\title{
LA CONTABILIDAD COMO SISTEMA COMPLEJO EN LAS ORGANIZACIONES: UNA NECESIDAD CONTRA-HEGEMÓNICA
}

\author{
A CONTABILIDADE COMO UM SISTEMA COMPLEXO NAS ORGANIZAÇÕES: UMA \\ NECESSIDADE CONTRA-HEGEMÔNICA
}

\author{
ACCOUNTING AS A COMPLEX SYSTEM IN ORGANIZATIONS: A COUNTER- \\ HEGEMONIC NEED
}

Milagros Elena RODRÍGUEZ ${ }^{1}$

RESUMEN: Las prácticas modernistas-coloniales de los especialistas de la Contabilidad se subordinan como antihumanas, en la mayoría de los casos, depredadoras del planeta-tierra y antiéticas; desde lo cual es urgente indisciplinar la Contabilidad tradicional, su reduccionismo. Con el proyecto transmodernista y transparadigma transcomplejo se realizó la investigación transmetódica con la deconstrucción rizomática, cumpliendo el objetivo complejo de analizar la Contabilidad como sistema complejo en las organizaciones como una necesidad contrahegemónica. En los rizomas reconstructivos, desde el des-ligaje de la decolonialidad y los sistemas hegemónicos en un re-ligaje inclusivo, decolonial, la Contabilidad con los grupos transdisciplinares, indisciplinando su propios estatutos; se renueva para cobrar nuevas fuerzas: nuevas maneras abiertas complejas, transdisciplinares, ecosóficas y antropolíticas para ejercer una tarea humana que no está de espalda al hacer en la humanidad urgente de civilizarse.

PALABRAS-CLAVE: Contabilidad. Complejidad. Organizaciones. Ecosofía.

RESUMO: As práticas colonialistas-modernistas dos especialistas em Contabilidade são subordinadas a anti-humanas, na maioria das vezes, predatórias do planeta-terra e antiéticas; da qual é urgente indisciplinar a Contabilidade tradicional, seu reducionismo. Com o projeto transmodernista e transcomplex do transparadigma, a pesquisa transmetódica foi realizada com desconstrução rizomática, atendendo ao complexo objetivo de analisar a Contabilidade como um sistema complexo nas organizações como uma necessidade contrahegemônica. Nos rizomas reconstrutivos, da desvinculação da descolonialidade e dos sistemas hegemônicos para uma re-vinculação inclusiva e descolonial, a Contabilidade com grupos transdisciplinares, indisciplina seus próprios estatutos; ela se renova para ganhar uma nova força: novos caminhos complexos, transdisciplinares, ecossóficos e antropoliticos abertos para realizar uma tarefa humana que não está dando as costas à humanidade, tornando-a urgente para se civilizar.

PALAVRAS-CHAVE: Contabilidade. Complexidade. Organizações. Ecosofia.

1 Universidad de Oriente (UDO), Cumaná - Venezuela. Docente Investigadora Postdoctoral Titular a Dedicación Exclusiva del Departamento de Matemáticas. Postdoctora en Ciencias de la Educación (UNEFA). ORCID: https://orcid.org/0000-0002-0311-1705. E-mail. melenamate@hotmail.com 
ABSTRACT: The modernist-colonial prácticos of the Accounting specialists are subordinated as anti-human, in most cases, predatory of the planet-earth and unethical; from which it is urgent to indiscipline traditional Accounting, its reductionism. With the transmodernist and transcomplex transparadigm project, the transmethodic research was carried out with rhizomatic deconstruction, fulfilling the complex objective of analyzing Accounting as a complex system in organizations as a counter-hegemonic need. In the reconstructive rhizomes, from the dis-linking of decoloniality and hegemonic systems into an inclusive, decolonial re-linking, Accounting with transdisciplinary groups, indiscipline its own statutes; it is renewed to gain new strength: new complex, transdisciplinary, ecosophic and anthropolitical open ways to exercise a human task that is not turning its back on humanity by making it urgent to civilize itself.

KEYWORDS: Accounting. Complexity. Organizations. Ecosophy.

En cualquier esquema ordenado, que le disponga un patrón a la vida de los seres humanos, hay que inyectar cierta dosis de anarquismo.

Bertrand Russell (2004).

Entre los problemas latentes en los que desangran con sus consecuencias, los seres humanos, países, y la tierra se encuentra sin duda la crisis de la economía mundial, la paradoja de vivir en pleno desarrollo en apariencia, en la era tecnológica, en plena globalización y que muchos mueran por la carencia de servicios tan elementales como comida, agua, y vivienda es la ironía en la cara de las políticas caducadas de quienes tiene el poder y lo han subvertido en autoritarismo.

Milagros Elena Rodríguez (2020a).

\section{Rizoma introito y transmetodología: el transmétodo de investigación la deconstrucción rizomática}

Los lemas Russell (2004) y Rodríguez (2020a) que presentan a la investigación, hablan de la subversión necesaria en los patrones fijos incambiables de conocer, ante el eminente fracaso de los poderes soslayantes; de allí que nos subvertimos ante al autoritarismo; y promovemos la libertad y con ello el fundamento de todo valor: la dignidad del ser humano. Para ello, la decolonialidad planetaria es nuestro norte; un Norte con el Sur y el Norte, con Occidente y todos los continentes; un permearse del respeto a toda forma de vida; hasta el planeta mismo.

El reduccionismo del proyecto modernista, que reduce a los seres humanos a objetos (MORÍN, 1999), ha llevado a que los políticos, jueces e individuos se reduzcan a sus más simples, cambiantes y radicales emociones psicológicas; más aún, que actúen como un productor de capital; sin tomar en cuenta su realidad compleja. Es por esto que para salir de la crisis o caos del trasfondo reduccionista y como estudia al hombre, la autora se apoyó en las 
teorías complejas y en su definición de la ética del hombre como individuo, sociedad y especie (MORÍN, 1999).

Las necesidades del momento, el desmontaje del sistema financiero mundial ante lo resultados es un hecho que ha sido deconstruido en los años sesenta (60) por el matemático Benoit Mandelbrot quien asevera que el comportamiento del sistema financiero es fractálico, y con ello lo es el de la Contabilidad, se rige no de manera lineal; hecho que ha tambaleado ante las evidencias reduccionista, han desmitificado a la Contabilidad como parte ese sistema. En ello nos unimos ante una teoría fractálica que sobre pasa la linealidad y nos anima a aportes esenciales y complejos del hacer de la Contabilidad, de su praxis.

El padre de esta teoría fractálica es Benoit Mandelbrot, en su libro Fractales $y$ Finanzas: una aproximación matemática a los mercados: arriesgar, perder y ganar, en donde estudia la relación que existe entre las matemáticas complejas y el mundo financiero, es quien da aportes para empezar a estudiar los sistemas financieros como un ciclo y una secuencia donde tarde o temprano todo se vuelve a repetir (MANDELBROT, 2006).

En particular, dentro y con el sistema financiero sabemos que las definiciones clásicas de la Contabilidad la declaran muchas veces como un sistema de información, y como tal está encargada "del registro, la elaboración y la comunicación de la información fundamentalmente de naturaleza económico financiera que requieren sus usuarios para la adopción racional de decisiones en el ámbito de las actividades económicas" (ALCARRIA, 2009, p. 8); mas esa declaración de sistema no la asumen como compleja. La Contabilidad no se encomienda sino sólo del sostenimiento de los registros contables, también presume el diseño de sistemas contables eficientes, la elaboración de previsiones, el examen de las transacciones previo al registro y la exegesis de la información.

Partimos, y estamos de acuerdo que la Contabilidad es base para decisiones gerenciales; pero que la gerencia junto al sistema económico y financiero ha arrastrado a la Contabilidad a una visión simplificada ajustada a un oficio; imposibilitándola de su comunicación con el resto de los sistemas; la han permeado de reduccionismo y el hacer de un oficio para responder al mercado globalizado actualmente.

En el marco de la teoría de la complejidad, no se puede dejar de lado la ciencia legado de la humanidad que la antecede, como lo es la matemática de orden fractálica como parte de una interpretación y una ramificación más amplia de lo que es la matemática euclidiana y su enfoque lineal del mundo económico (MANDELBROT, 2006). Las matemáticas fractálicas son necesarias para estudiar el comportamiento de curvas y movimientos que tienen lugar todos los días en el campo de las finanzas, en la Contabilidad. 
Según Quezada (2006), se puede aseverar que al emprender trabajar con la teoría de la complejidad, las ideas se tropezaron con una gran tenacidad al cambio, por parte de los sectores más moderados de la ciencia y aún hoy hay algunos investigadores que exponen oposición y aprensión a la gran presencia de la no-linealidad en los comportamientos en la naturaleza y al caos como una consecuencia genérica de ella. Y la Contabilidad forma parte de esa resistencia, de ese ejercicio de poder.

Los métodos de investigación bajo el paradigma modernista han sido motivo de crítica y la puesta en evidencia de sus insuficiencias en las investigaciones tradicionales tales como: reduccionismo, determinismo, saberes soterrados execrados, entre otras (RODRÍGUEZ, 2019). En esta investigación transcompleja, que confirmamos es conjunción compleja y transdisciplinar y va en contra de la hegemonía reduccionista y parcelada ella tiene realización fuera del proyecto modernista-postmodernista-colonial; la transmodernidad como cosmovisión "episteme transfigura el entorno científico, cultural, económico, político, social y gerencial; es decir donde se desarrolla una determinada acción humana que propicie el cambio" (GUZMÁN, 2014, p. 128). Así, en el marco de este transparadigma, de acuerdo con la deconstrucción rizomática como transmétodo se analiza la Contabilidad como sistema complejo en las organizaciones desde una necesidad contra-hegemónica como objetivo complejo de la investigación.

Ejemplificamos la connotación compleja y transdisciplinar de la transcomplejidad como un acto subversivo ante la denominación de: introducción, desarrollo, resultados y conclusiones de las investigaciones tradicionales; y vamos a complejizarlos en un ir y venir con los rizomas; denotaciones de la Biología que indica que el tallo va a la raíz, a las hojas y estas vuelven a la raíz siempre en comunicación con el todo. En el rizoma "no hay un comienzo real sino en el medio. Allí donde la palabra "génesis" recupera plenamente su valor etimológico de "devenir", sin relación con un origen" (ZOURABICHVILI, 2007, p. 95). Por ello, el discurso rizomático es complejo y no obedece a estructuras fijas estáticas.

Vamos a ver en el discurso "la multiplicidad característica propia de una perspectiva rizomática, ya que esto implica que no hay un método y menos un camino único, por lo cual no se puede "descalificar a priori". Son muy importantes estas referencias sobre el rizoma, ya que estimulan un pensamiento de la multiplicidad, en cuanto rechazo de un punto de origen" (SICERONE, 2017, p. 91); de allí que esta libertad que no es libertinaje investigativo en la construcción no atiende a juicios modernistas-postmodernistas-coloniales, tiene su plena realización en la decolonialidad planetaria; por donde nos pasearemos regresando a los puntos del rizoma; por ejemplo a la crisis; pero no nos quedamos allí, pues vamos a una 
reconstrucción esperanzadora de la Contabilidad como sistema complejo; al final regresando a la crisis dejaremos futuras investigaciones insinuadas de continuidad de la línea de investigación.

La autora en dicho transmétodo es sujeto investigador activo con sus subjetividades y experiencia en las líneas de investigación que convergen a la continuación de indagaciones. En este caso esta indagación se ubica en la línea de investigación: economía-administracióngestión-y finanzas transcomplejas.

\section{Rizoma: la crisis de la Contabilidad como sistema reduccionista en las organizaciones deterministas}

La Contabilidad tradicional responde al sistema capitalismo y a las estructuras organizativas de la empresa que de este emergen como formas de racionalización, esto explica por qué la Contabilidad recurrió a la invención de la partida doble, que se adscribe como herramienta matemática de aprehensión de la realidad y que aún se mantiene (IRAUSQUÍN, 2015). Esa realidad ha sido sobre pasada en la crisis cuando de manera general estudiamos la crisis de los sistemas financieros mundiales, y desmotamos la regularidad matemática que han venido usando; su linealidad ha fracasado ante la complejidad eminente del sistema. Es así como, es urgente por ejemplo, en un subsistema de los sistemas financieros, clamar por economistas transcomplejos como estrategas con principios ecosóficos y antropoéticos (RODRÍGUEZ, 2020a). De la misma manera este estudio alude a la Contabilidad.

El mundo de las finanzas quiere proteger bajo la erige de la tecnología, sus sistemas; ¿qué pasa cuando los sistemas que se crean para proteger vienen de la mano con perpetradores que pueden violentarlos? "Bajo su égida, la globalización, el desarrollo, la occidentalización, tres caras del mismo fenómeno, se han mostrado incapaces de tratar los problemas vitales de la humanidad” (MORÍN, 2011, p. 78). ¿Qué ha aportado a la humanidad en su problemática mundial, que es la civilización y su crisis la Contabilidad? ¿Ha salido de su parcela para realizar aportes al respecto?

El propósito básico de la Contabilidad es proveer de información útil acerca de una entidad económica, para suministrar la toma de decisiones de sus diferentes usuarios; bien sean estos accionistas, acreedores, inversionistas potenciales, clientes, administradores, gobierno, pero también de sistemas secretos de inteligencia y de las compañías mundiales dominantes de la historia de la humanidad; en efecto, como la Contabilidad le sirve a un conjunto diverso de beneficiarios, así se ocasionan diversos subsistemas. 
Veamos, de acuerdo con las diferentes necesidades de la concepción reduccionista de la complejidad, con base en las diferentes necesidades de información de los distintos segmentos de usuarios, la información total que se genera en una organización económica se estructura en tres subsistemas: el subsistema de información financiera que lleva la Contabilidad financiera, el subsistema de información fiscal con la Contabilidad fiscal y el subsistema de información administrativa con la Contabilidad administrativa (GUAJARDO; ANDRADE, 2005). Estos subsistemas se deben interrelacionar; deben ser considerados transdisciplinarmente con los aportes de las ciencias; su epistemología debe quebrantarse; e ir a las fronteras de dichos subsistemas y debilitar su pensamiento abismal, a fin de nutrirlos con el aporte del resto de los sistemas del conocer de los saberes.

Es de hacer notar, que la Contabilidad en su fundamente tradicional siempre ha sido una organización, supuesta, formal, algorítmica, de exegesis del entorno, que discurre sobre una genealogía; su historia y filosofía. Algunos autores trabajan en una futura Contabilidad líquida, que sea su antípoda, sin genealogía, será un mapa abierto, conectable en todas sus dimensiones y direcciones, "desmontable, alterable, susceptible de recibir constantemente modificaciones, que conecta eslabones semióticos de información, organizaciones reticulares y en la que noción de unidad sólo aparecerá cuando se produzca en una multiplicidad de circunstancias relacionadas" (MARTÍNEZ, 2008, p. 22). Son intentos de desmontaje de la crisis de la Contabilidad y sus fundamentos; pero la lucha es contra gigantes de las finanzas que asumen su razón de pérdida y ganancia al riesgo del sistema de vida del planeta.

En contraste a las miradas hegemónicas de lo ambiental, que como ya se ha visto han pretendido poner el ambiente en las lógicas del capital, reduciéndolo a un factor potencializador del crecimiento, globalización o erradamente el desarrollo; como sinónimos de la misma crisis, han surgido nuevas perspectivas, en las cuales se deconstruye la noción de ambiente hegemónica y se edifica una nueva desde la complejidad de la realidad (QUINTERO; RAMÍREZ, 2016).

En ese sentido el tocar dichos cimentos soslayadores; es urgente tocar la formación del contador, ¿Quién es el contador como ciudadano ante el mundo? Pareciera que el contador al igual que todos los profesionales con formación modernista-postmodernista-colonial ejercen sus funciones siéndole leal al sistema capitalista; desmitificando las consecuencias; así su ejercicio es de un ciudadano antiético, antipolítico que rompe con la misma concepción de ciudadano del mundo; aquel que se des-liga de sus viejas prácticas hegemónicas.

Queremos definir el des-ligaje y re-ligaje como necesidad para avanzar a la decolonialidad y como necesidad de ir a transepistemes en el proyecto transmoderno. Para 
cubrir esas necesidades e intentar incluirlas siempre como posibilidades de mejoras hacen falta un re-ligar como urgencia de la transmodernidad (RODRIGUEZ, 2019b) se trata de desligarse de los viejos patrones soslayadores de hacer contabilidad e ir a la decolonialidad planetaria como posibilidad de convivir los saberes científicos y los complejos que van emergiendo en la reconstrucción del conocimiento en general.

El re-ligar des-ligando primeramente, puede hacernos ver cómo es necesario darnos cuenta de que la verdad única nadie la tienen, y que sin embargo, no por ello el poder biopolítico del momento pierde oportunidad para ejercer su conveniencia en las falsas políticas contables; que se disfrazan de decoloniales (RODRÍGUEZ, 2020b).

El sistema de Contabilidad constituye el conjunto de elementos o componentes que interactuando de forma coordinada proporciona información y control útil para la toma de decisiones gerenciales en una empresa; esa es su generalidad de funcionalidad. Este sistema opera y es parte del complejo compuesto de elementos que conforman a una organización u empresa, así se observa que de la misma manera en que las organizaciones cambian en su estructura modernista para perfeccionar sus patrones mundiales, formas de relacionarse interna y externamente y de operar en función de la perdida y la ganancia, de la maximización de la producción, del dominio hegemónico; así ha cambiado el sistema de Contabilidad, ya que éste opera con la información y datos provenientes de todas las unidades que efectúan, directa o indirectamente, transacciones financieras, y hacia las mismas debe proporcionar información y control a la medida de sus necesidades y procesos de cambio (DE NOBREGA, 2011).

Así, es notorio este cambio, no ha obedecido a la concepción compleja, a la función ética política de los ciudadanos contadores; ni a las necesidades que se imponen y se develan; las necesidades de la crisis mundial de la economía, que ha permeado todos los sistemas; y ha puesto a prueba la ignominia en la formación del contador indiferente ante tales crisis. Se trata de deconstruir en la Contabilidad

Su dominio conceptual está dado por los conocimientos sistematizados que la Contabilidad presenta respecto de su ontología (la necesidad de controlar la riqueza para ordenar el sistema económico, con criterio metodológico y operativo de racionalidad financiera), una gnoseología pragmática y prescriptiva (basada en el conocimiento empírico) con normas que determinan objetos informativos que se usan en los procesos decisorios y un ethos de la aplicación práctica bajo ciertas expectativas de equidad social (GIL, 2020, p. 96). 
Sabemos que el desmontaje de la Contabilidad tradicional tiene otras aristas a ser tratadas y entramadas; que las seguiremos estudiando en lo que viene, pero también comenzaremos a reconstruir con las subjetividades de la autora, derecho inclusivo del transmétodos que le da al sujeto investigador, que aporta en primera persona su análisis.

\section{Rizoma reconstrucción: la Contabilidad como sistema complejo en las organizaciones como una necesidad contra-hegemónica}

Estamos en la reconstrucción como parte de la deconstrucción rizomática. La Contabilidad, entendida como sistema de información y como elemento de una acción comunicativa, debe lograr, que toda información sea germen de comunicación; esto es que la acción comunicativa sea satisfecha, atendiendo los elementos: a) de producir un mensaje racional, b) de códigos semejantes, c) expresiva de la interioridad del emisor y aceptada por la interlocución como cierta, en razón de la posibilidad de interacción del receptor (HABERMAS, 1987). ¿Se cumple esa realidad en la Contabilidad actual?

Cabe destacar que, la Contabilidad como sistema complejo que está inmerso en los sistemas financieros y económicos debe considerar que las matemáticas fractálicas son las responsables de anular la falsa creencia de que el mercado financiero era un sistema lineal y continuo siempre en crecimiento y de aportar a los estudios de la economía la premisa de que los sistemas económicos tienen comportamientos con diversas formas, y da la propiedad de ciclo a los sistemas financieros. De allí, "los sistemas de análisis actuales no sirven, y que la única manera de entender el funcionamiento del sistema financiero es utilizando la teoría del caos y los fractales" (MANDELBROT; HUDSON, 2006, p. 102). Por ello, la consideración de la complejidad en la teoría del caos y los aportes de los estudios de fractales es urgente en la Contabilidad como sistema complejo emergente.

La Contabilidad debe ir a concebir una política del ser humano en el planeta, política de la responsabilidad tierra-patria (RODRÍGUEZ, 2020a), "política multidimensional, pero no totalitaria. El desarrollo de los seres humanos, de sus relaciones mutuas, del ser societal, constituye el propósito mismo de la política del hombre en el mundo, que apunta a la persecución de la hominización” (MORÍN, 1993, p. 166). Esa política multidimensiona examina de manera responsable en ejercicio como ciudadano del profesional de la contabilidad; le anida un ejercicio ético como ciudadano del mundo. Es ese uno de los principales re-ligajes urgentes de construir bajo la conciencia de responsabilidad de lo urgente de nuestros aportes. 
Se trata de un ejercicio de servicio a la humanidad desde el ejercicio de la Contabilidad; este ejercicio va examina las experiencias históricas dispares, particularmente de los grupos dominados, marcadas por profundas desigualdades sociales y económicas, discriminación socio-racial, violencia y las dinámicas de exclusión propias del sistema Occidental-Norte dominante perpetuados en la globalización; se trata de afrontar la humanidad en su inhumanidad. Los grupos de contadores también forman parte de los subalternos que modelan formas de subjetividad política y memoria social; ellos deben autosocorrerse en su decolonialidad evaluado su propia praxis; sus dinámicas soslayadoras y su conformidad como ser humano en la tierra.

Esa regeneración moral-ética urgente desde pensares antropolíticos para por reconocer que no se trata de un orden moral excluyente favorecedor del sistema dominante; no se trata de una profunda inclusión que se fundamentó en generar un estancamiento consciente e inconsciente de la colectividad. Se trata de un avivamiento en la que los excluidos se ven tomado en cuenta en su potencialidad.

Se debe ir a un des-ligar y re-ligar de la Contabilidad (RODRÍGUEZ, 2019b) modernista-colonial, el proyecto modernista los invita, los retrae de su caducado oficio; es realizar un escudriñamiento de la cientificidad de la Contabilidad, donde se debe apostar al ejercicio colaborativo transdisciplinar de profesionales; que vayan a deconstruir el peligroso proyecto disciplinar contable centrado tan sólo en la mirada científica, bajo los desarrollos que definió la modernidad.

La Contabilidad entonces con los grupos transdisciplinares, indisciplinando su propios estatutos se renueva para cobrar nuevas fuerzas; nuevas maneras abiertas complejas, transdisciplinares, ecosóficas y antropolíticas para ejercer una tarea humana que no está de espalda al hacer en la humanidad antihumana. Si, los contadores entonces van a imbricarse en una tarea de las tres ecologías que conforman la ecosofía: la ecología social, espiritual y ambiental (GUATTARI, 1996); donde la Contabilidad tiene mucho que aportar.

¿Cómo se incluye como necesidad urgente esa sabiduría complejizada de habitar en el planeta: la ecosofía en la Contabilidad? La Contabilidad en un dialogo transdisciplinar, en las civilizaciones puede provocar un estado de conciencia de nuestro accionar en el mundo, las necesidades de identidad tanto individual como planetaria, para saber vivir y convivir desde el amor por nuestra creación en una sola tierra-patria; con conocimiento pertinentes; la formación para la toma de decisiones adecuadas; es inclusión es urgente.

Se debe des-elitizar, re-ligar, des-ligar con las disciplinas conjuncionándolas, indisciplinando las disciplinas; rompiendo sus fronteras fuera del pensamiento abismal que las 
separa, la responsabilidad social del docente como ciudadano, y la esencia misma de ser humano; una transversalizándola a los saberes de la Contabilidad; que son simplemente y grandemente saberes del mundo y para el mundo. Es motivo de la continuación de la línea de investigación provocamos la inclusión ecosófica en las transepistemologías con los campos ecosóficos, para futuras investigaciones: esto es: científico, emocional, práctico y espiritual. Así, las concepciones de la ecosofía son el puente unitivo de los saberes en general donde siempre la matemática tiene su aporte; hagamos realidad este sentido en la Contabilidad bajo concepciones complejas.

¿Bajo concepciones ecosóficas cuáles son los retos de la complejidad compleja? Los retos son dignos de asumirse; en tanto su caducada epistemología se ahoga en la indiferencia ante los problemas que los arropan; rompe la Contabilidad el reducido pensar cuantitativo e ir a penar en lo cualitativo, sociocrítico, rizomáticamente en una transgestión; más allá de la tradicionalidad; enriqueciéndola de configuraciones dinámicas del conocer; es la gestión de información y control bajo nuevos representaciones de pensamiento en organizaciones sociales-políticas complejas.

En lo cualitativo, es urgente desmitificar la noción de números en la contabilidad; pues este nos significa sólo cantidad cuantitativa; es una relación de transcendencias sociales, jurídicas, económicas, políticas, ecológicas que proceden del carácter de producción; y que de tomarse en cuenta estarían dando paso a la matemáticas de la complejidad y la transdisciplinariedad de sus concepciones a los fractales; así como indicamos pasa con los números en los sistemas contables.

Para ello, la Contabilidad como sistema complejo re-liga tareas en "el nuevo modo de pensar se enfrenta a lo divergente, convergente, desconstrucción, fragmentación, heterogeneidad, desterritorialización, codificación, descodificación, lo uno y lo múltiple, en fin, a la complejidad" (UGAS, 2008, p. 37). Se trata de una tarea titánica, de conciencia ecosófica de habitar en el planeta bajo la responsabilidad social. El principal reto para los contadores, para el contralor en ejercicio y sus propia critica, es merecidamente conseguir una configuración en el ejecutar que "considere conceptos, ideas y principios de la complejidad, la no linealidad, lo cambiante, lo dinámico, el caos, la incertidumbre; es el reconocimiento de la organización como sistema complejo y del sistema de Contabilidad como parte del mismo" (DE NOBREGA, 2011, p. 55).

La desterritorialización en la contabilidad como sistema complejo es urgente; su territorio de números en invadido por la regularidad de las ciencias; y el contador va a ellas a complejizarse en sus transgestiones de cálculos, funciones; análisis. Ella, la Contabilidad 
compleja, al desterritorializarse engrandece sus propios territorios; no abandona su funcionalidad; por el contrario la engrandece; amplia el lente de su mirada y se compromete con el re-ligaje permanente.

Por ello, la conformación en materia de matemáticas de la complejidad, la complejidad estudios transdisciplinares y complejos son esenciales en el contador en formación; no tendrán una salida importante, sino decolonizan su propia tarea como ejercicio de primerísimo des-ligar (RODRIGUEZ, 2019b). En ello, los acercamientos transdisciplinares permitirán a los especialistas ir más allá de sus propias aristas de miradas transdisciplinares; puede colaborar efectivamente con otras ciencias; e ir al abrazo de los saberes.

Es de hacer notar, que el aporte de las nuevas teorías, como la complejidad, las de la informática las develaciones coloniales aportan sustantivos esenciales a la complejidad para que su lucha contra-hegemónica sea exitosa con serios avances; es así como

El dominio conceptual de la Contabilidad marcha hacia la elaboración de hipótesis sobre cuestiones socioambientales relativas a la sustentabilidad tanto como sobre la productividad global; su dominio epistémico interno se encamina hacia nuevas metodologías como mediciones cualitativas, el balance social integral y el cuadro de valor agregado; el dominio epistémico derivado pone sus intereses no tanto en las Finanzas como en la Sociología, la Economía política y la Ecología (GIL, 2020, p. 96).

En tal sentido, embicándonos en conceptos de la ciencia compleja Contabilidad vemos que su concepción compleja llevaría a reconocer bienes y servicios ambientales, dejando la idea de precio monetario y emergen en valores de atributos y características de no-mercado. El la ecología social de la Contabilidad compleja puede ayudar a contribuir con soluciones más integrativas y decoloniales en un marco de la transdisciplinariedad frente a las demandas sociales de responsabilidad cognitiva-afectiva de la humanidad y de las tecnologías.

\section{Rizoma conclusivo: la Contabilidad como sistema complejo en un devenir urgente}

Con el proyecto transmodernista y transparadigma transcomplejo se realizó la investigación transmetódica con la deconstrucción rizomática, con el objetivo complejo de analizar la Contabilidad como sistema complejo en las organizaciones como una necesidad contra-hegemónica. Ubicada en la línea de investigación: economía-administración-gestión-y finanzas transcomplejas. Ha venido dando sus frutos y se sigue en el ardor de la indagación.

Pensar en la Contabilidad como sistema complejo en las organizaciones como una necesidad contra-hegemónica, es pensar en el des-ligaje y re-ligaje en la praxis que nos lleva a 
evaluarnos y en la búsqueda de rezagos coloniales que nos hacen resistirnos al cabio, a la confrontación contra-hegemónica necesaria ante las mutaciones de los sistemas actuales con nuevos artefactos de soslayaciones.

El re-ligaje ecosófico propende variantes del ciudadano Contador que emergen en medio de los sistemas complejos: un antropolítico por excelencia que le da pertenencia a su noble tarea; pero enmarañado con los saberes del mundo, por la cualidad del número, la cantidad de sus riesgos; y la complejización de las necesidades a tender en el convulsionado mundo del conocer tronchado por interés capitalistas inhumanos.

Si se trata de no repetir los mismos errores que parecen ser la norma ante el obedecer al sistema depredador de vidas, no sólo humanas; la vida del planeta. Con el transmétodos la deconstrucción rizomática hemos intentado dar una primera mirada compleja a la contabilidad desde el transmétodos; ya hemos citado investigaciones que erigen el sistema complejo en la contabilidad; la diferencia es la marcada necesidad de la decolonialidad; no podemos sembrar en el mismo terreno infértil de la colonialidad; allí no podemos germinar transepistemes para para contabilidad.

Seguimos en el ardor de la decolonialidad buscando anheladas complejidades de aportes de la línea: economía-administración-gestión- y finanzas transcomplejas. Continuamos en ello, contando con mentes ávidas de cambio, en la comprensión de que el sujeto investigador no puede seguirse execrado de las investigaciones; por ello el transmétodo es excelente en el rescate y la salvaguarda de las esencias principales de todo ser humano: su afectividad, subjetividades de responsabilidades ante el planeta tierra: como lo aspiramos de los contadores complejos.

AGRADECIMENTOS Y DEDICATORIA: Reconociendo la creación de Dios en cada sentir de su autora, que atiende al amor como su primera excelsitud heredada del Padre Dios amado, me despido dándole las gracias por su infinita sabiduría; donde siempre la gloria es de Dios en el nombre de Jesucristo. Así, "Y sobre todas estas cosas vestíos de amor, que es el vínculo perfecto" (Colosenses 3:14), "porque Jehová da la sabiduría, Y de su boca viene el conocimiento y la inteligencia" (Proverbios 2:6). Por ello debemos estar atentos a meditar la palabra de Dios: “no os acumuléis tesoros en la tierra, donde la polilla y la herrumbre destruyen, y donde ladrones penetran y roban" (Mateo 6:19). 


\section{REFERENCIAS}

ALCARRIA, J. Contabilidad financiera I. Paris: Departament de Finances i Comptabilitat, 2009.

DE NOBREGA, F. El sistema de Contabilidad en la complejidad de las organizaciones. Actualidad Contable FACES, Mérida, n. 22, p. 45-56, 2011.

GIL, M. Determinismo de la Contabilidad en la complejidad: hipótesis introductoria.

Ciencias Administrativas, Buenos Aires, n. 16, p. 91-98, 2020. DOI

https://doi.org/10.24215/23143738e 068

GUAJARDO, G.; ANDRADE, N. Contabilidad para no contadores. México: McGRAWHILL/Interamericana Editores, S.A. de C.V. 2005.

GUATTARI, F. Las tres ecologías. Madrid: Pre-Textos, 1996.

GUZMÁN, J. Cosmovisión emergente de la naturaleza de la realidad desde la perspectiva transcompleja. In: Tecnología y transcomplejidad. Caracas: Red de Investigadores de la transcomplejidad REDIT, Universidad Bicentenaria de Aragua, 2014.

HABERMAS, J. Teoría de la acción comunicativa. Madrid: Ediciones Taurus, 1987.

IRAUSQUÍN, C. Aproximación a la visión compleja de los términos: Contabilidad, ambiente y desarrollo. MUL TICIENCIAS, Punto fijo, v. 15, n. 1, p. 12-18, 2015.

MANDELBROT, B.; HUDSON, R. Fractales y finanzas: una aproximación matemática a los mercados: arriesgar, perder y ganar. Nueva York: Tusquets Editores, 2006.

MARTÍNEZ, G. De la Contabilidad sólida a la Contabilidad líquida: ¿crisis o muerte de la factualidad? In: SIMPOSIO NACIONAL DE INVESTIGACIÓN CONTABLE Y

DOCENCIA, 7., 2008, Popayán. Anais [...]. Popayán, Colombia: Universidad del Cauca, 2008. Disponible en: http://guillomartinezpino.jimdo.com/textos-del-autor/ Consultado en: 10 dic. 2020.

MORÍN, E. Entrevista con Edgar Morín, análisis del libro “Con la cabeza bien puesta”. ORUS, la revue, v. 1, p. 1-4, 1999.

MORÍN, E. La Vía para la salvación de la humanidad. Madrid: Espasa Libros, 2011.

MORÍN, E. Tierra-patria. Barcelona: Kairós, 1993.

QUEZADA, A. Fractales y opinión pública: una aplicación del exponente de Hurst al estudio de la dinámica de la identificación ideológica. 2006. Tesis (Doctorado) - Facultad de Psicología, Universidad de Barcelona, Barcelona, 2006.

QUINTERO, A.; RAMÍREZ, A. Por caminos contra-hegemónicos hacia una contabilidad ecológica. Contaduría Universidad de Antioquia, Antioquia, v. 68, p. 287-312, 2016. 
RODRÍGUEZ, M. E. Deconstrucción: un transmétodo rizomático transcomplejo en la transmodernidad. Sinergias educativas, Ecuador, v. 4, n. 2, p. 43-58, 2019a.

RODRÍGUEZ, M. E. Re-ligar como práctica emergente del pensamiento filosófico transmoderno. ORINOCO Pensamiento y Praxis, Ciudad Bolívar, v. 11, p. 13-3, 2019b.

RODRÍGUEZ, M. E. Transcomplex economists: strategies with ecosophic and antropoetic principles. Rev. Sem Aspas, Araraquara, v. 9, n. 1, p. 4-26, jan./jun. 2020a. DOI: https://doi.org/10.29373/sas.v9i1.13993

RODRÍGUEZ, M. E. La ecología de los saberes en la educación matemática decolonial transcompleja. RCEF: Rev. Cien. Foco Unicamp, Campinas (SP), v. 13, e020015, 2020b.

RUSSELL, B. Sceptical essays. 2. ed. New York: Routledge Classics, 2004.

SICERONE, D. Rizoma, epistemología anarquista e inmanencia en la filosofía de deleuze y guattari. Revista de Filosofía, Maracaibo, v. 87, p. 83-94, 2017.

UGAS G. La complejidad, un modo de pensar. Caracas: Ediciones del Taller permanente de estudios epistemológicos en Ciencias Sociales, 2008.

ZOURABICHVILI, F. El vocabulario deleuze. Buenos Aires: Ediciones Atuel, 2007.

\section{Cómo referenciar este artículo}

RODRÍGUEZ, M. E. La contabilidad como sistema complejo en las organizaciones: una necesidad contra-hegemónica. Rev. Sem Aspas, Araraquara, v. 10, e021003, jan./dez. 2021. e-ISSN 2358-4238. DOI: https://doi.org/10.29373/sas.v10i00.14552

Enviado el: $13 / 12 / 2020$

Aceptado el: 04/03/2021

Publicado el: $30 / 06 / 2021$ 